\title{
La prévention de la grippe aujourd'hui
}

\author{
S. Zimmerli, K. Mühlemann \\ pour la Commission d'épidémiologie de la Société suisse \\ d'infectiologie*
}

\begin{abstract}
Résumé
Causée par le virus influenza, la grippe représente depuis des siècles un facteur important de morbidité et de mortalité. Des stratégies préventives et thérapeutiques sont mises en œuvre au sein de la population pour répondre aux épidémies saisonnières annuelles, d'importance diverse, et au risque de pandémie. En Suisse, l'élaboration (comme dans d'autres pays) d'un plan de pandémie national, puis d'une ordonnance à ce sujet (actuellement en consultation), la campagne de l'Office fédéral de la santé publique (OFSP) pour la prévention de la grippe - qui s'étendra sur plusieurs années et concerne déjà la saison à venir - et les nouvelles substances thérapeutiques (inhibiteurs de la neuraminidase) et nouveaux vaccins disponibles ont permis d'examiner la question sous de nouveaux aspects.
\end{abstract}

Les médecins praticiens jouent un rôle important dans la prévention de la grippe. Ils contribuent de manière essentielle à augmenter le taux de vaccination, notamment au sein des différents groupes à risque. Durant la période grippale, ils ont, en outre, la tâche importante de reconnaître et d'annoncer les cas de grippe, ainsi que les complications inhabituelles de la vaccination. Le corps médical joue également un rôle clé à la veille d'une pandémie et durant celle-ci, comme le souligne le plan élaboré à cet effet.

Dans cet article, la Commission d'épidémiologie de la Société suisse d'infectiologie (SSI) donne un aperçu de la situation actuelle en matière de préven-

\footnotetext{
* Membres de la Commission d'épidémiologie:

Dominique Blanc, André Burnens, Patrick Francioli, Christoph Hatz, Kathrin Mühlemann, Didier Pittet, Pierre-A. Raeber, Robert Steffen, Nicolas Troillet, Rainer Weber, Andreas Widmer
}

\section{Correspondance:}

Dr Kathrin Mühlemann, p.-d.

Institut des maladies infectieuses

de l'Université de Berne

Friedbühlstrasse 51

CH-3010 Berne

E-mail: kathrin.muehlemann@ifik.unibe.ch tion grippale. Plutôt que d'entrer dans les détails, elle renvoie sciemment le lecteur à différentes sources. Certaines questions n'étant pas encore réglées pour l'instant, une suite à cet article est prévue afin de tenir le corps médical informé des derniers événements.

\section{Introduction}

Maladie infectieuse connue depuis des siècles, la grippe constitue, aujourd'hui encore, un facteur important de morbidité et de mortalité. Hormis les épidémies saisonnières annuelles, le risque d'une nouvelle pandémie (épidémie à l'échelle mondiale) existe toujours. Pour faire obstacle à la grippe et aux complications qu'elle engendre, d'importantes nouvelles stratégies ont été adoptées et de nouveaux moyens de prévention et de traitement ont été développés ces dernières années. En Suisse, comme dans d'autres pays, un groupe d'experts a élaboré un plan d'action dans l'éventualité d'une pandémie grippale. Un projet d'ordonnance a suivi, qui est actuellement en consultation. Une campagne nationale vient d'être lancée pour promouvoir la vaccination antigrippale des personnes appartenant aux différents groupes à risque (personnes de 65 ans et plus, malades chroniques, etc.) et du personnel médical et paramédical. En ce qui concerne les virostatiques développés récemment, la question se pose de savoir dans quelle mesure ils peuvent être utilisés judicieusement dans la pratique. Quant au nouveau vaccin par voie nasale, il semble encore poser quelques problèmes de sécurité vaccinale. Des informations complètes sont largement disponibles en la matière ou seront publiées sous peu. Il reste, cela dit, plusieurs questions ouvertes, qui devraient recevoir une réponse - du moins partielle - dans les prochaines semaines ou d'ici quelques mois.

\section{Bases}

Importance de l'influenza

Dans le langage populaire, toute infection des voies respiratoires est une "grippe». Toutefois, contrairement aux autres infections virales respiratoires, la vraie grippe, celle causée par les virus de la famille influenza, évolue de façon plus brutale et peut être à l'origine de complications graves, voire létales (p. ex. des pneumonies). Chaque année dans notre pays, entre 100000 et 300000 consultations médicales, entre 1000 et 5000 hospitalisations et pas moins de 400 à 1000 décès sont dus à l'épidémie de grippe hivernale (http://www.bag.admin.ch/aktuell/ f/PandemieVo/pandemie.htm, [1]). Ces décès concernent surtout des personnes de plus de 65 ans, des patients déjà atteints de maladies cardio-pulmonaires et des enfants en bas âge. Chez les jeunes adultes, une part importante de l'incapacité de travail est à mettre au compte de la grippe.

Le patient qui se remet d'une grippe acquiert une immunité sous la forme d'anticorps contre les anti- 
gènes viraux spécifiques de surface, l'hémagglutinine et la neuraminidase. Ceux-ci changent toutefois régulièrement de structure ("antigenic drift" ou dérive antigénique), échappant ainsi à la pression de la sélection naturelle au sein de la population. Ainsi, en 2 à 3 ans habituellement, des souches se développent, qui sont suffisamment différentes pour pouvoir réinfecter d'anciennes victimes. La dérive antigénique est également la raison pour laquelle le vaccin contre la grippe doit être adapté aux nouveaux virus en circulation et répété chaque année.

A des intervalles imprévisibles, de nouvelles sortes de virus influenza apparaissent dont les antigènes de surface présentent de grandes différences avec ceux des souches habituellement en circulation ("antigenic shift» ou saut antigénique). Si ces virus se transmettent facilement d'une personne à l'autre, il peut en résulter une pandémie d'influenza. Comme la majeure partie de la population n'est pas immunisée contre cette nouvelle souche de virus, un bien plus grand nombre de personnes peuvent tomber gravement malades que lors d'une épidémie habituelle de grippe. Ainsi, en 1918-1919, lors de la plus grave pandémie connue jusqu'ici, plus de 20 millions de personnes sont décédées [2, 3]. En Suisse seulement, un quart de la population tomba malade et près de 25000 personnes moururent (http://www.bag.admin. ch/aktuell/f/PandemieVo/pandemie.htm).

L'organisation mondiale de la santé (OMS) surveille l'activité de la grippe par le biais d'un réseau de centres régionaux étendu au monde entier. Grâce aux informations ainsi récoltées, il est possible de déterminer chaque année la composition du vaccin de la saison suivante. La surveillance de l'activité grippale en Suisse se fonde sur le recensement des cas de suspicion de grippe dans le cadre du système de déclaration volontaire Sentinella [4]. A partir de prélèvements (nez et gorge) effectués par les médecins de Sentinella, le Centre national de la grippe, à Genève, isole et identifie les souches virales d'influenza. Les résultats sont transmis à l'OMS, à l'EUROGROG et à l'OFSP et publiés sous forme de bilans hebdomadaires durant la saison de la grippe (http://www. influenza.ch/).

Inhibiteurs de la neuraminidase

La neuraminidase des virus influenza permet aux virus néoformés de se libérer des récepteurs à la surface de la cellule hôte. Si cet enzyme est inhibé, les virus restent liés à la cellule et ne peuvent donc plus en infecter d'autres. Les inhibiteurs de la neuraminidase bloquent la partie active de cette enzyme de manière spécifique. Or, malgré un taux élevé de mutations, cette partie est quasiment identique chez les virus de type $A$ et $B$, si bien que les inhibiteurs de la neuraminidase sont efficaces contre tous les virus influenza (y compris les éventuelles souches pandémiques). Deux préparations sont actuellement utilisées; on les distingue principalement par leur mode d'administration différent: le Relenza ${ }^{\circledR}$ (zanamivir) est pris en inhalation ( 2 doses à $5 \mathrm{mg}, 2 \times$ par jour) et le Tamiflu ${ }^{\circledR}$ (oseltamivir), par voie orale (1 capsule de $75 \mathrm{mg}, 2 \times$ par jour). Ces deux médicaments doivent être administrés dans les 24 heures après l'apparition des premiers symptômes pour avoir l'effet escompté. Tous deux peuvent diminuer la gravité et la durée des symptômes de grippe ainsi que les complications causées par l'influenza; de plus, ils sont en général bien tolérés [5]. Les deux sont également efficaces en prophylaxie et peuvent p. ex. être administrés après une vaccination contre la grippe jusqu'à l'apparition d'une réponse immunitaire suffisante, afin de protéger la personne vaccinée contre une infection. Toutefois, le Tamiflu ${ }^{\circledR}$ n'est pas enregistré en Suisse pour une application à titre préventif, même si son efficacité en prophylaxie a été démontrée pour les épidémies de grippe dans les homes pour personnes âgées. Les deux médicaments coûtent environ Fr. 80.- pour une thérapie de 5 jours ou un traitement préventif de 10 jours et sont à la charge du patient. Les inhibiteurs de la neuraminidase ne remplacent pas le vaccin contre la grippe. Lors de l'épidémie annuelle, ils peuvent être administrés au patient lorsque les signes cliniques correspondent à ceux d'une grippe et que le patient a consulté le médecin dans les 24 heures après l'apparition des premiers symptômes. En cas de pandémie, ils constituent, jusqu'à l'arrivée d'un vaccin, les médicaments les plus importants aussi bien pour la prévention que pour le traitement.

\section{Vaccins contre la grippe}

\section{Vaccins administrés par voie parentérale}

Les vaccins contre la grippe contiennent habituellement trois souches de virus influenza: deux de type A et une de type B. La composition du vaccin est modifiée chaque année selon les recommandations de l'OMS. Ces dernières années, les vaccins à particules entières, contenant le virus entier sous forme inactivée, ont été largement remplacés par des vaccins de type "split" (constitués de fragments de virus) ou "subunit" (ne contenant que les antigènes de surface hémagglutinine et neuraminidase). Une caractéristique de ces derniers est qu'ils sont mieux supportés, notamment par les enfants. Les vaccins dits virosomaux contiennent les antigènes de surface liés à une membrane de phospholipides (liposomes). Ils se caractérisent par une meilleure stimulation des anticorps, mais coûtent également plus cher. Selon différentes études (non contrôlées), l'effet protecteur des vaccins antigrippaux est, chez les personnes de plus de 65 ans, de 37 à 64\% [6, 7]. Quant à l'effet protecteur contre les complications d'une grippe dans ce groupe d'âge, il est à peu près du même ordre. Les frais de vaccination des groupes à risque (voir tableau) sont pris en charge par les caisses-maladie. De plus amples informations sur la vaccination contre la grippe sont contenues dans les références [7, 8].

Pour la saison grippale 2000/2001, un vaccin entièrement nouveau a fait son apparition en Suisse, sous la forme d'un spray nasal (Nasalflu ${ }^{\circledR}$ ). Il s'agit en principe d'un vaccin de type "subunit», virosomal, contenant également une toxine d'Escherichia coli 
thermostable comme adjuvant. Contrairement aux vaccins conventionnels, administrés par injection intramusculaire, ce vaccin nasal se distingue par l'induction d'anticorps spécifiques locaux IgA dans la muqueuse nasale. Bien que l'on ne dispose pour l'instant que de très peu d'informations sur son effet protecteur, il semble que ce vaccin soit au moins équivalent à ceux administrés par voie parentérale. Le nouveau mode d'administration par le nez représente un progrès technologique important et prometteur dans le domaine du développement de nouveaux vaccins. L'application d'un vaccin sur les muqueuses devrait, d'une part, aboutir à un meilleur effet protecteur grâce à l'immunité de la muqueuse induite localement et, d'autre part, mettre fin à certains problèmes logistiques liés à l'administration parentérale des vaccins. L'extension de la technologie aux vaccins universellement recommandés, tels que celui contre la rougeole, pourrait représenter un pas de géant dans le domaine de la prévention des maladies infectieuses.

Cela dit, les inconvénients de ce premier vaccin nasal contre la grippe sont: un taux relativement élevé d'effets indésirables (écoulement nasal, nez bouché, éternuements, etc.), la nécessité d'une administration en deux fois (à intervalle d'environ une semaine) et un prix relativement élevé (environ Fr. 60.par dose), qui - pour l'instant - n'est pas pris en charge par les caisses. L'observation de quelques cas de paralysie faciale chez des personnes ayant reçu le vaccin nasal au cours de la saison grippale 2000/2001 a créé un doute quant à l'existence possible d'un lien causal. Cette question très grave est restée sans réponse pour le moment, d'où les incertitudes au sein du corps médical, de la population et de la presse. Il est difficile d'apprécier la situation, notamment du fait que le nombre de cas observés jusqu'ici est malgré tout restreint et que l'on ne dispose, en Suisse, d'aucuns relevés prospectifs et permanents permettant une comparaison avec la fréquence de base des paralysies faciales (indépendamment d'une vaccination). De tels chiffres ont été collectés rétrospectivement ces derniers mois par l'Institut de médecine sociale et préventive de l'Université de Zurich et on a, en outre, procédé à une étude de cas-témoins. D’autres études sont prévues. Les résultats des études déjà en cours ne sont pas encore disponibles pour l'instant. Attendus pour cet automne, ils constitueront une base de décision importante pour la question de l'admission du vaccin nasal pour la saison de vaccination $2001 / 2002$.

La campagne nationale de prévention de la grippe de l'Office fédéral de la santé publique

Pour améliorer constamment la couverture vaccinale antigrippale dans les groupes à risque et au sein du personnel médical et paramédical, l’OFSP a planifié et lancé, en collaboration avec les cantons, le corps médical et d'autres responsables dans ce domaine, une campagne nationale de prévention de la grippe qui s'étendra sur plusieurs années.
En se fondant sur le nombre de doses de vaccin distribuées, on a pu estimer le taux de vaccination de la population à environ $6 \%$ en 1996. Ce taux est ensuite monté jusqu'à 14\% en l'an 2000 (comparable aux chiffres de l'Allemagne, de l'Angleterre et de la Belgique [9]). Il faut savoir cependant que, dans notre pays, le taux de vaccination des groupes à risque (voir texte encadré) et du personnel médical diffère certes selon les régions, mais reste dans l'ensemble insuffisant (Ammon 1998). Une enquête effectuée au printemps 2001, sur mandat de l'OFSP, auprès des personnes âgées de toute la Suisse a montré, chez les plus de 65 ans, un taux moyen de vaccination de 48,0\%, le taux étant plus élevé en Suisse romande $(53,1 \%)$ qu'en Suisse italienne $(47,5 \%)$ et alémanique $(46,4 \%)$. Les campagnes de vaccination contre la grippe menées dans les différents cantons romands constitueraient une explication plausible de ces différences [10].

L'intention de la campagne nationale est donc de sensibiliser au thème de la grippe les personnes particulièrement menacées et concernées (voir texte encadré) et d'élever considérablement la couverture vaccinale. Celle-ci devra monter jusqu'à $60 \%$ chez les personnes de 65 ans et plus, d'ici à 2005. On vise même un taux de $70 \%$ dans les autres groupes à risque du point de vue médical. Un taux comparable serait également souhaitable chez le personnel médical et paramédical en contact avec les groupes à risque. Différentes études ont montré que les médecins praticiens jouent un rôle primordial dans l'information des patients à risque sur le vaccin contre la grippe et dans leur décision de se faire vacciner [11]. Cette année, la campagne de vaccination de l'OFSP a pour mot d'ordre: "La grippe arrive. Le vaccin est là» ("Grippe? Ich bin geimpft»; «influenza, ti ho fregato. Mi sono vaccinato"). Les informations et documents concernant cette campagne peuvent être obtenus auprès du Centre de documentation sur la prévention de la grippe, 3000 Berne 6 (tél. 031 35214 60; fax 03135214 71).

La campagne de prévention de la grippe est également - bien qu'indirectement - d'une importance capitale pour le plan pandémique. Plus on utilise de vaccins contre la grippe et plus les fabricants peuvent se permettre d'en augmenter la production. Ce qui, sur le plan logistique, ne manquerait pas d'influer positivement sur la préparation de grandes quantités de vaccin en cas de (menace de) pandémie. En outre, grâce à une meilleure connaissance des problèmes liés à la grippe et des possibilités de prévention, la population serait, elle aussi, mieux préparée à une telle catastrophe.

\section{Préparation à une éventuelle pandémie}

\section{Le plan en cas de pandémie}

On peut s'attendre à ce que, dans le futur, de nouvelles souches de virus influenza déclenchent des pandémies. Or, dans notre pays également, une telle pandémie, avec son taux élevé de morbidité et de 
mortalité, tournerait à la catastrophe. Afin d'établir les mesures à prendre dans de telles circonstances pour pallier la pénurie aiguë de vaccins et de médicaments antigrippaux, un plan a été élaboré par le groupe de travail Influenza mis sur pied par le Département fédéral de l'intérieur (http://www. admin.ch/bag/aktuell/f/PandemieVo/pandemie.htm, groupe de travail Influenza) [12]. Par l'élaboration et la mise en place de mesures appropriées, la préparation à une pandémie vise en premier lieu à réduire le nombre de décès prématurés et à limiter la morbidité au sein de la population lors d'une telle occurrence. Partant d'une analyse de situation concernant la surveillance, la prévention et le contrôle de la grippe en Suisse, le plan pandémique explique d'une part comment assurer des soins de santé suffisants, même dans des conditions difficiles. D'autre part, il décrit les démarches à entreprendre pour maintenir le fonctionnement de la vie publique et la sécurité interne et externe du pays. Il contient également des recommandations pour se préparer de façon optimale à une éventuelle pandémie, notamment quant à l'amélioration de la capacité de production et d'approvisionnement du vaccin et à l'augmentation des stocks de médicaments antiviraux.

Une information complète de la population est également très importante pour éviter les réactions de panique. En même temps, il s'agit de tenter de parvenir à un consensus social sur la manière de répartir selon des critères éthiques un bien limité (vaccins, médicaments), en accordant la priorité aux groupes les plus menacés. Le plan en cas de pandémie représente les éléments de base proposés par les experts pour se préparer à une pandémie; il est périodiquement adapté en fonction des nouvelles connaissances acquises.

\section{L'ordonnance sur la pandémie}

Se fondant sur le plan en cas de pandémie, le Département fédéral de l'intérieur (DFI) a conclu qu'il fallait, aujourd'hui déjà, prendre des mesures en prévision d'une pandémie. Un projet d'ordonnance a donc été élaboré en tenant compte des éléments proposés par les experts. Ce projet se trouve actuellement en procédure de consultation (le texte et les explications peuvent être consultés sur le site de l'OFSP, sous www.bag.admin.ch/, en français, allemand, italien et anglais). Il attribue au DFI et à l'OFSP la compétence de prendre des mesures avant, pendant et après une pandémie de grippe afin d'en atténuer les conséquences éventuelles. Le projet d'ordonnance comprend, d'une part, des dispositions visant à promouvoir la prévention générale d'une pandémie d'influenza et, d'autre part, des mesures à prendre en cas de menace aiguë de pandémie. Les bases juridiques sont: la loi sur les épidémies, qui donne au Conseil fédéral les compétences en matière de lutte contre les maladies transmissibles, et la loi sur l'approvisionnement du pays, qui contient une base législative pour garantir l'approvisionnement en médicaments en cas de grave pénurie. La procédure de consultation a donné lieu à un large débat sur la problématique de la pandémie grippale, débat auquel la FMH a également participé en donnant son avis. Tout le monde s'accorde sur la nécessité de prendre des mesures préparatoires appropriées. En ce moment, différents aspects financiers du projet d'ordonnance sont analysés.

\section{Le rôle du médecin praticien}

Le plan pandémique souligne le rôle déterminant que les médecins praticiens ont à jouer dans l'amélioration de la couverture vaccinale de la population. Durant la saison grippale, ils ont en outre la fonction importante de reconnaître et d'annoncer les cas de grippe (notamment dans les institutions) ainsi que les complications inhabituelles survenant après une vaccination. En cas de pandémie, le plan recommande de laisser les généralistes, internistes, pédiatres et spécialistes en ORL continuer à soigner et, le cas échéant, à vacciner leurs patients à leur cabinet, et de recourir éventuellement aux spécialistes d'autres disciplines pour mener des campagnes de vaccination. Dans ce contexte, le plan de pandémie prévoit également des mesures pour soutenir le praticien, tant sur le plan stratégique que professionnel.

\section{Remerciements}

La commission souhaite remercier les personnes suivantes pour leur soutien et leur collaboration à la rédaction du texte et pour leur lecture attentive et critique du manuscrit: Mme Catherine Ammon, responsable de la campagne de prévention de la grippe à l'OFSP, le Dr H. H. Brunner, président de la FMH, le Dr H. C. Matter, chef de la section «Maladies virales et système Sentinellan à l'OFSP et le Prof. M. Täuber, président de la Société suisse d'infectiologie.

\section{Références}

1 Egger M, Jennings S, Spuhler Th, Zimmermann HP, Paccaud F, Somaini B. Sterblichkeit während Grippeepidemien in der Schweiz 1969-1985. Schweiz Med Wochenschr 1989;119: 434-9.

2 Ambrosch F, Fedson DS. Influenza vaccination in 29 countries. An update to 1997. Pharmacoeconomics 1999;16 Suppl 1: 47-54.

3 Ammon CE. La promotion en Suisse de la prophylaxie de la grippe chez les personnes courant un risque accru de complications en cas d'affection grippale. Genève: Institut de médecine sociale et préventive, Université de Genève; Office fédéral de la santé publique; 1998

4 Groupe de travail Influenza. Pandémie de grippe: Analyse et recommandations pour la Suisse - Un rapport du groupe de travail Influenza (plan en cas de pandémie).

5 Armstrong GL, Conn LA, and Pinner RW: Trends in Infectious Disease Mortality in the United States During the 20th Century. JAMA 1999;281:61-6.

6 Birrer A, Bodmer T, Bollag U, Cloetta J, Egli N, Flückiger H, et al. Sentinella 1998. Annual report of the Swiss Sentinel Surveillance Network. Jahresbericht des schweizerischen Sentinella-Meldesystems. Rapport annuel du système de déclaration Sentinella en Suisse. Berne: Schriftenreihe Sentinella-Jahresberichte, Office fédéral de la santé publique; 2000 . 
7 Office fédéral de la santé publique, groupe de travail Influenza, Commission suisse pour les vaccinations. Recommandations pour la prévention de la grippe. Dans: Office fédéral de la santé publique (éd.). Maladies infectieuses. Diagnostic et prévention. Berne: Office fédéral de la santé publique, Suppl. XIII; 2000. Ce document est accessible sur l'internet (http://www.bag.admin.ch/infekt) prev/f/index.htm); il peut également être obtenu auprès de l'Office fédéral de la santé publique, Division épidémiologie et maladies infectieuses, case postale, 3003 Berne (tél: 03132387 06; fax: 03132387 95).

8 Gauthey L, Toscani L, Schira JC. Vaccination contre la grippe en Suisse. Soz Präventivmed 1997;42:107-111.
9 Glezen WP. Emerging infections: Pandemic Influenza. Epidemiol Rev 1996;18:64-76.

10 Gross PA, Hermogenes AW, Sacks HS, Lau J, Levandowski RA. The efficacy of influenza vaccine in elderly persons A meta-analysis and review of the literature. Ann Intern Med 1995;123:518-27.

11 Gubareva LV, Kaiser L, Hayden FG. Influenza virus neuraminidase inhibitors. Lancet 2000;355:827-35.

12 Wood JJ. Prevention and treatment of influenza. N Engl J Med 2000;343:1778-87.

\section{Tableau 1}

Recommandations de l’Office fédéral de la santé publique pour la prévention de la grippe.

La vaccination contre la grippe sera effectuée de préférence entre mi-octobre et mi-novembre. L'Office fédéral de la santé publique recommande une vaccination annuelle, en particulier pour les groupes à risque suivants:

personnes de plus de 65 ans.

Adultes et enfants exposés à des risques accrus de complications en raison de maladies graves. Ce groupe comprend les personnes qui souffrent de maladies cardiaques ou pulmonaires chroniques, d'asthme bronchique, de malformations cardiaques congénitales, de fibrose kystique, de troubles métaboliques chroniques (notamment le diabète), d'insuffisance rénale, d'hémoglobinopathie ou d'immunosuppression.

Adultes et enfants qui requièrent un suivi médical régulier et personnes qui ont été hospitalisées au cours de l'année.

Résidents de homes ou de maisons de retraite, pensionnaires d'institutions pour malades chroniques.

Personnel médical et soignant ainsi que toute personne en contact direct avec les patients et/ou résidents des hôpitaux et cliniques, des cabinets médicaux, des services de soins à domicile, des homes pour personnes âgées et des établissements de soins ou de cure.

Pour plus d'informations, voir: Office fédéral de la santé publique, groupe de travail Influenza, Commission suisse pour les vaccinations. Recommandations pour la prévention de la grippe. Dans: Office fédéral de la santé publique (éd.). Maladies infectieuses. Diagnostic et prévention. Berne: Office fédéral de la santé publique, Suppl. XIII; 2000. Le document est accessible sur l'internet (http://www.bag.admin.ch/infekt/prev/f/index.htm)

\section{Tableau 2}

Le plan en cas de pandémie en Suisse.

Mesures proposées par les experts pour la prévention d’une pandémie.

Est périodiquement adapté aux découvertes les plus récentes.

Indique:

- les mesures à prendre pour réduire la mortalité et la morbidité au sein de la population en cas de pandémie

- la façon d'assurer des soins de santé suffisants dans des conditions difficiles

- les démarches à entreprendre pour maintenir le fonctionnement de la vie publique et la sécurité intérieure et extérieure du pays

Contient des recommandations pour une préparation optimale à une éventuelle pandémie, par exemple:

- Amélioration de la capacité de production de vaccin et de l'approvisionnement en vaccin

- Augmentation des stocks de médicaments antiviraux

L'ordonnance sur la pandémie

- Offre la base juridique nécessaire pour régler les mesures à prendre avant, pendant et après une pandémie d'influenza afin d'en atténuer les conséquences éventuelles.

Le texte et les explications concernant le plan pandémique et l'ordonnance peuvent être consultés sur le site internet de l'OFSP (www.bag.admin.ch/), en français, allemand, italien et anglais. 


\section{Tableau 3}

Des nouveaux médicaments antiviraux contre la grippe (inhibiteurs de la neuraminidase).

\begin{tabular}{|c|c|}
\hline Substances & Relenza $^{\circledR}$, par voie nasale, à l’aide d’un inhalateur («diskhaler»), et Tamiflu ${ }^{\circledR}$, par voie orale. \\
\hline Spectre d'action & contre tous les virus influenza A et B. \\
\hline Efficacité & $\begin{array}{l}\text { administré dans les } 24 \text { heures après l'apparition des symptômes, ces médicaments dimi- } \\
\text { nuent leur gravité et leur durée ainsi que les complications. }\end{array}$ \\
\hline Sécurité & bonne tolérance. \\
\hline Frais & $\begin{array}{l}\text { environ Fr. 80.- pour un traitement de } 5 \text { jours ou une prophylaxie de } 10 \text { jours. Les frais sont à la charge du } \\
\text { patient. }\end{array}$ \\
\hline
\end{tabular}

\section{Tableau 4}

La campagne de prévention de la grippe de l'Office fédéral de la santé publique.

La campagne nationale de prévention de la grippe lancée par l'OFSP, en collaboration avec les cantons, le corps médical et d'autres responsables dans ce domaine, s'étendra sur plusieurs années; elle a pour but de sensibiliser au sujet de la grippe les personnes particulièrement menacées ou concernées et d'augmenter notablement la couverture vaccinale d'ici à 2005, à savoir: jusqu'à

$60 \%$ chez les personnes de 65 ans et plus;

$70 \%$ chez les autres groupes à risque du point de vue médical;

$70 \%$ chez le corps médical et les soignants.

Les médecins praticiens jouent un rôle primordial dans l'information des patients à risque et dans leur décision de se faire vacciner contre la grippe.

Le slogan de la campagne de vaccination de cette année est: «La grippe arrive. Le vaccin est là»; «Grippe? Ich bin geimpft»; «Influenza, ti ho fregato. Mi sono vaccinato".

D'autres informations ainsi que des documents concernant cette campagne peuvent être obtenus auprès du Centre de documentation sur la prévention de la grippe, 3000 Berne 6 (tél. 03135214 60; fax 03135214 71).

\section{Tableau 5}

Vaccins contre la grippe administrés par voie parentérale: portrait.

\begin{tabular}{|c|c|c|}
\hline Composition & \multicolumn{2}{|c|}{$\begin{array}{l}\text { vaccins inactivés contenant } 3 \text { types de virus influenza ( } 2 \text { souches de type A, } 1 \text { de type B) sous forme de par- } \\
\text { ticules virales entières ou fragmentées ou de sous-unités (ces derniers pouvant aussi être obtenus sous forme } \\
\text { de vaccins de type "virosomal»). }\end{array}$} \\
\hline \multirow[t]{2}{*}{ Efficacité } & personnes > 65 ans & 30 à $65 \%$ \\
\hline & adultes en bonne santé & 68 à $90 \%$ \\
\hline \multirow[t]{2}{*}{ Effets indésirables: } & réaction locale & $\begin{array}{l}\text { chez } 20 \text { à } 40 \% \text { des patients vaccinés; érythème, douleurs, prurit; réaction le plus } \\
\text { souvent légère et de courte durée ( } 2 \text { jours); }\end{array}$ \\
\hline & réaction systémique & $\begin{array}{l}<5 \% \text { des patients vaccinés; symptômes grippaux (fièvre, myalgies, nausées, etc.) } \\
\text { pouvant persister } 1 \text { à } 2 \text { jours (plus fréquents avec les vaccins à particules virales } \\
\text { entières). }\end{array}$ \\
\hline
\end{tabular}

